\title{
Full photon statistics of a light beam transmitted through an optomechanical system
}

\author{
Andreas Kronwald, ${ }^{1,}{ }^{*}$ Max Ludwig, ${ }^{1}$ and Florian Marquardt ${ }^{1,2}$ \\ ${ }^{1}$ Friedrich-Alexander-Universität Erlangen-Nürnberg, Staudtstr. 7, D-91058 Erlangen, Germany \\ ${ }^{2}$ Max Planck Institute for the Science of Light, Günther-Scharowsky-Straße 1/Bau 24, D-91058 Erlangen, Germany
}

(Received 16 March 2012; published 31 January 2013)

\begin{abstract}
In this paper, we study the full statistics of photons transmitted through an optical cavity coupled to nanomechanical motion. We analyze the entire temporal evolution of the photon correlations, the Fano factor, and the effects of strong laser driving, all of which show pronounced features connected to the mechanical backaction. In the regime of single-photon strong coupling, this allows us to predict a transition from sub-Poissonian to super-Poissonian statistics for larger observation time intervals. Furthermore, we predict cascades of transmitted photons triggered by multiphoton transitions. In this regime, we observe Fano factors that are drastically enhanced due to the mechanical motion.
\end{abstract}

DOI: 10.1103/PhysRevA.87.013847

PACS number(s): 42.50.Ar, 07.10.Cm, 42.50.Lc, 42.65.-k

\section{INTRODUCTION}

Photon statistics serves as a powerful tool in quantum optical experiments. For example, it has been used to identify the strong coupling between radiation and an atom by observing nonclassical photon antibunching [1] and multiphoton transitions [2-4]. While all of these examples show how photon statistics can be influenced by coupling to internal degrees of freedom, only a few experiments have demonstrated the effects of any kind of mechanical motion. These mostly involve the classical motion of atoms flying through cavities [5-8]. A first step into the quantum domain has been reported in [9] by showing the impact of quantized ionic motion onto the photon statistics. In all of these examples, however, the statistics is heavily influenced by both internal and external degrees of freedom. We will analyze a setup where, in contrast to those systems, the photon correlations (both on short and long time scales) are entirely due to the coupling of photons to a single degree of freedom, namely a quantum mechanical vibrating resonator.

These cavity optomechanical systems [10] offer a new domain to study the effects of quantized mechanical motion on the statistics of photons, without the need for atom trapping. The field has been developing rapidly during the past few years. It is driven by the goals of probing the quantum motion of nanomechanical devices, implementing ultrasensitive measurements, and exploiting the light-mechanics coupling for quantum information processing, as well as answering fundamental questions about quantum mechanics. Recently, the mechanical degree of freedom was cooled down close to its quantum ground state using optomechanical sideband cooling $[11,12]$. Thus, it now becomes important to analyze probes for the quantum dynamics of these systems. Experimentally, the most straightforward access is provided by the light field.

Earlier works $[27,28]$ studied the linearized dynamics of small fluctuations in the context of homodyne detection, where the light field was described by continuous variables and squeezing effects were predicted. A particularly powerful tool is given by the statistics of single photons, as those are very sensitive to interactions and reveal the temporal correlations produced by the dynamics. A first study in this direction

\footnotetext{
*andreas.kronwald@physik.uni-erlangen.de
}

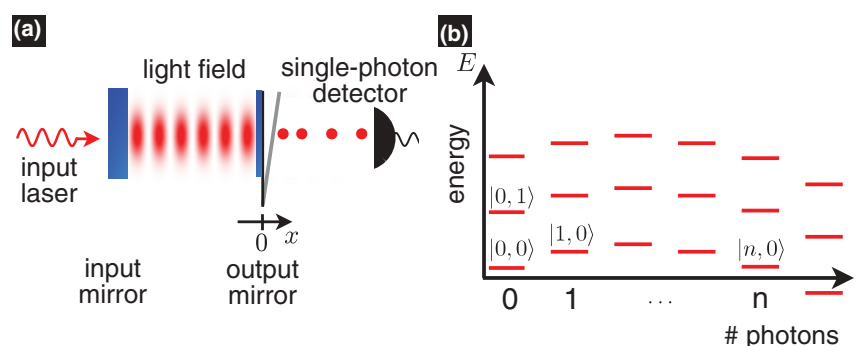

FIG. 1. (Color online) (a) The standard optomechanical setup, where transmitted photons are detected by a single-photon detector. As we will show, the strong backaction of the mechanical motion onto the light field dynamics leads to new features in the photon correlations. (b) Nonlinear level scheme induced by the lightmechanics coupling. The eigenstates read $\left|n_{a}, n_{b}\right\rangle$, where $n_{a}\left(n_{b}\right)$ denotes the photon (phonon) number.

recently led to the prediction of photon blockade [18] in optomechanical systems. Here, we will take a significant step further by analyzing the full statistics of the stream of transmitted photons. In contrast to [18], our numerical approach allows us to study many new aspects, including the regime of strong laser driving, the full temporal structure of photon correlations, the Fano factor, and higher moments of the distribution of transmitted photons.

All of these properties become particularly interesting in the regime of single-photon strong light-mechanics coupling, which (although challenging) is now being approached experimentally [11-16]. In this regime, the single photon coupling rate becomes comparable to the photon decay rate, and a few remarkable phenomena have already been predicted. A "classical to quantum crossover" may be observed in nonlinear optomechanical dynamics [17], and photon blockade effects [18], non-Gaussian [19], and nonclassical [20] mechanical states may be produced. Additionally, diverse Schrödinger-cat states may be generated [21,22], multiple cooling resonances may be observed [23], and certain dark states may exist [24]. Furthermore, QND photon and phonon readout and nonlinearities could be enhanced in appropriate two-mode setups $[25,26]$.

This paper is organized as follows: First, we introduce the optomechanical model in Sec. II, and show how photons and phonons can be decoupled formally. In Sec. III, we define the Lindblad master equation for the optomechanical system 
to describe its dissipative dynamics. Then we discuss the quantum jump trajectory technique giving access to the full counting statistics, which is described thereafter. Furthermore, we introduce the Fano factor as a measure for the photon statistics and connect it to the two-photon correlation function $g^{(2)}(\tau)$. At the end of this section, we show how the Fano factor is influenced by finite detector efficiencies. In Sec. IV A we discuss a weakly driven, strongly coupled optomechanical system, with emphasis on the full time dependence of the photon correlator. In Sec. IV B, we present our main results in the regime of strong driving, where we introduce and discuss the regime of photon cascades.

\section{THE MODEL}

We consider a generic optomechanical setup consisting of a laser-driven optical cavity, where one of the end mirrors is attached to a vibrating resonator [see Fig. 1(a)]. The coherent dynamics is described by the Hamiltonian,

$$
\hat{H}=-\hbar \Delta \hat{a}^{\dagger} \hat{a}-\hbar g_{0}\left(\hat{b}^{\dagger}+\hat{b}\right) \hat{a}^{\dagger} \hat{a}+\hbar \Omega \hat{b}^{\dagger} \hat{b}+\hbar \alpha_{L}\left(\hat{a}^{\dagger}+\hat{a}\right),
$$

written in a frame rotating at the laser frequency $\omega_{L}[10,29]$. Here, $\hat{a}(\hat{b})$ is the photon (phonon) annihilation operator, $\Delta=$ $\omega_{L}-\omega_{\text {cav }}$ is the laser detuning from resonance, and $g_{0}$ is the single-photon optomechanical coupling constant. $\Omega$ denotes the frequency of the mechanical resonator, and $\alpha_{L}$ is the laser driving amplitude.

Photons and phonons can be decoupled by applying the socalled polaron transformation $[21,22] \hat{\tilde{H}}=\hat{U}^{\dagger} \hat{H} \hat{U}$ to Eq. (1), where $\hat{U}=\exp \left[g_{0} \hat{a}^{\dagger} \hat{a}\left(\hat{b}^{\dagger}-\hat{b}\right) / \Omega\right]$. This yields

$\hat{\tilde{H}}=-\hbar \Delta \hat{a}^{\dagger} \hat{a}+\hbar \Omega \hat{b}^{\dagger} \hat{b}-\hbar \frac{g_{0}^{2}}{\Omega}\left(\hat{a}^{\dagger} \hat{a}\right)^{2}+\hbar \alpha_{L}\left(\hat{a}^{\dagger} \hat{D}^{\dagger}+\right.$ H.c. $)$

where the mechanical displacement operator $\hat{D}=$ $\exp \left[g_{0}\left(\hat{b}^{\dagger}-\hat{b}\right) / \Omega\right]$ now enters the transformed driving term. The elimination of the photon-phonon coupling gives rise to a photon-photon interaction term. This leads to an anharmonic photon energy level scheme, cf. Fig. 1(b), which drastically influences the intracavity photon statistics if $g_{0}^{2} \gtrsim \kappa \Omega$ [18].

\section{METHODS}

\section{A. Dissipative dynamics}

The dissipative dynamics of the open optomechanical system is described by a Lindblad master equation,

$$
\begin{aligned}
\dot{\hat{\rho}}= & \frac{i}{\hbar}[\hat{\rho}, \hat{H}]+\kappa_{I} \mathcal{D}[\hat{a}] \hat{\rho}+\Gamma_{M}\left(n_{\mathrm{th}}+1\right) \mathcal{D}[\hat{b}] \hat{\rho} \\
& +\Gamma_{M} n_{\mathrm{th}} \mathcal{D}\left[\hat{b}^{\dagger}\right] \hat{\rho}-\frac{\kappa_{O}}{2}\left\{\hat{a}^{\dagger} \hat{a}, \hat{\rho}\right\}+\kappa_{O} \hat{a} \hat{\rho} \hat{a}^{\dagger},
\end{aligned}
$$

where $\hat{\rho}$ is the density matrix for the photons and phonons. $\kappa_{I}\left(\kappa_{O}\right)$ denotes the photon loss rate through the input (output) mirror, where $\kappa=\kappa_{I}+\kappa_{O}$ is the total cavity decay rate and $\Gamma_{M}$ is the phonon decay rate. Furthermore, $n_{\text {th }}^{-1}=\exp \left(\hbar \Omega / k_{B} T\right)-1$, where $T$ is the mechanical bath temperature. The Lindblad superoperator reads $\mathcal{D}[\hat{a}] \hat{\rho}=$ $\hat{a} \hat{\rho} \hat{a}^{\dagger}-\left\{\hat{a}^{\dagger} \hat{a}, \hat{\rho} / 2\right\}$, where $\{\hat{a}, \hat{b}\}=\hat{a} \hat{b}+\hat{b} \hat{a}$.

\section{B. Quantum jump trajectories}

In experiments, the photon statistics can be analyzed by measuring the times when a photon leaves the cavity through the output mirror, cf. Fig. 1(a). To simulate this detection process, we use quantum jump trajectories [30-34], which can be understood as an "unraveling" of the master equation (3). It has recently been employed to discuss single-phonon detection in optomechanical systems [35].

If a photon is detected ("photon jump") during a time interval $[t, t+\delta t]$ with probability $p_{j}=\eta \kappa_{O}\left\langle\hat{a}^{\dagger} \hat{a}\right\rangle(t) \delta t$, the information about the system is updated according to

$$
\hat{\rho}(t+\delta t)=\frac{\hat{a} \hat{\rho}(t) \hat{a}^{\dagger}}{\operatorname{Tr}\left[\hat{a}^{\dagger} \hat{a} \hat{\rho}(t)\right]} .
$$

Here, $\eta$ is the photon detector efficiency, i.e., the ratio of the number of detected photons to the total number of transmitted photons.

If no photon is detected, $\hat{\rho}$ evolves according to Eq. (3) [where the original Hamiltonian (1), without polaron transformation, is used in our numerics], with the last term of Eq. (3) replaced by $(1-\eta) \kappa_{O} \hat{a} \hat{\rho} \hat{a}^{\dagger}$. After each such time step, the state has to be normalized again. This evolution describes the increase of our knowledge due to the absence of a detection event $[32,36]$.

Overall, we obtain stochastic traces of photon detection events which directly correspond to what would be observed in an experiment that registers single photons. This is one of the principal advantages of employing the quantum jump trajectory method. From these trajectories, we gain access to the full statistics, including arbitrary moments and the complete time dependence of photon correlations.

\section{Full counting statistics}

The full counting statistics $p\left(N, T_{S}\right)$ [38] is the probability of measuring $N$ transmitted photons in a time interval $T_{S}$, for a constant detection rate $\dot{\bar{N}}=\eta \kappa_{O} \bar{n}$. Here, $\bar{n}$ is the steady-state photon number of Eq. (3). The fluctuations of the detected photon number can be characterized via the Fano factor,

$$
\mathcal{F}_{\mathrm{c}}\left(T_{S}\right)=\left(\left\langle N^{2}\right\rangle-\langle N\rangle^{2}\right) /\langle N\rangle,
$$

where $\left\langle N^{m}\right\rangle=\sum_{N=0}^{\infty} N^{m} p\left(N, T_{S}\right)$. If $\mathcal{F}_{\mathrm{c}}\left(T_{S}\right)<1$ there is sub-Poissonian statistics (on that observation time scale), whereas for $\mathcal{F}_{\mathrm{c}}\left(T_{S}\right)>1$ the photons obey super-Poissonian statistics.

The long-time limit of the Fano factor yields the zerofrequency shot noise power, $S_{I I}[\omega=0]=\dot{\bar{N}} \mathcal{F}_{\mathrm{c}}(\infty)$. Thus, $\mathcal{F}_{c}(\infty)$ can be interpreted as the effective number of detected photons during a single transmission process (i.e., during a single "bunch" of photons).

\section{Photon correlations $g^{(2)}(\tau)$ and connection to the Fano factor}

Another measure of photon correlations is given by the two-photon correlation function

$$
g^{(2)}(\tau)=\frac{\left\langle\hat{a}^{\dagger}(0) \hat{a}^{\dagger}(\tau) \hat{a}(\tau) \hat{a}(0)\right\rangle}{\left\langle\hat{a}^{\dagger}(0) \hat{a}(0)\right\rangle^{2}} .
$$

Here we have assumed that a steady state exists, and all expectation values in this definition are taken with respect 
to the steady-state density matrix of the full Lindblad master equation (3).

The two-photon correlation function $g^{(2)}(\tau)$ can be interpreted as a measure for detecting a photon at time $\tau$ conditioned on the detection of a photon at time $\tau=0$. Using $g^{(2)}(\tau)$ we can distinguish three regimes [37]:

(i) If $g^{(2)}(\tau)=1$ for all delay times $\tau$, the photon detection events are statistically independent and obey Poissonian statistics.

(ii) Photon bunching is defined via $g^{(2)}(0)>1$. In this case, there is a tendency that photons arrive in groups.

(iii) The photons are antibunched when $g^{(2)}(0)<1$, which is a pure quantum effect. In contrast to photon bunching, the transmitted photons tend to avoid each other.

The Fano factor $\mathcal{F}_{\mathrm{c}}$ defined in Sec. III C is connected to this two-photon correlation function $g^{(2)}(\tau)$ via [38]

$$
\mathcal{F}_{\mathrm{c}}\left(T_{S}\right)=1+\dot{\bar{N}} \int_{-T_{S}}^{T_{S}} d \tau\left(g^{(2)}(|\tau|)-1\right)\left(1-|\tau| / T_{S}\right)
$$

Thus, for small sampling times $T_{S}$, we can distinguish photon antibunching and bunching using $\mathcal{F}_{c}\left(T_{S}\right)$ : If the photons are antibunched, $g^{(2)}(0)<1$ and, hence, the slope of the Fano factor $\mathcal{F}_{c}\left(T_{S}\right)$ is negative for small sampling times. In the case of photon bunching, however, $g^{(2)}(0)>1$, such that $\mathcal{F}_{c}\left(T_{S}\right)$ has a positive slope for small $T_{S}$.

Note that photon antibunching does not always correspond to sub-Poissonian photon statistics, which has already been shown for fluorescent photons [39]. We will show that also for the optomechanical system, antibunching and sub-Poissonian statistics do not necessarily coincide, cf. Fig. 3. Furthermore, photon bunching need not correspond to super-Poissonian statistics [40].

\section{E. Dependence of the Fano factor on the detector efficiency $\eta$}

The Fano factor $\mathcal{F}_{c}\left(T_{S}\right)$ depends on the detector efficiency $\eta$. The relation

$$
\mathcal{F}_{c}\left(T_{S}\right)=\frac{\mathcal{F}_{c}^{\text {all }}\left(T_{S}\right)+\left(\kappa-\eta \kappa_{O}\right) / \eta \kappa_{O}}{1+\left(\kappa-\eta \kappa_{O}\right) / \eta \kappa_{O}}
$$

connects the measured Fano factor $\mathcal{F}_{c}$ to $\mathcal{F}_{c}^{\text {all }}$. Here, $\mathcal{F}_{c}^{\text {all }}$ is the Fano factor that would be obtained when monitoring all decay channels with an ideal photon detector. Thus, we can reconstruct the ideal measurement outcome even if the output ports are observed with a nonideal detector. Therefore, without loss of generality, we will assume $\eta=1$ in the following.

\section{RESULTS}

\section{A. Weak laser drive}

We start by considering a weak drive such that the photon number inside the cavity remains small, $\left\langle\hat{a}^{\dagger} \hat{a}\right\rangle \ll 1$. In contrast to previous results [18], we are able to discuss the photon correlations $g^{(2)}(\tau)$ at arbitrary time delays, which is crucial to capture the long time scales induced by the mechanical motion [9].

Figures 2(a)-2(c) show a typical trajectory in the photonblockade regime [18], where the mechanical bath temperature is assumed to be zero. The intracavity photon number $\left\langle\hat{a}^{\dagger} \hat{a}\right\rangle$ decreases due to a photon jump. This behavior indicates

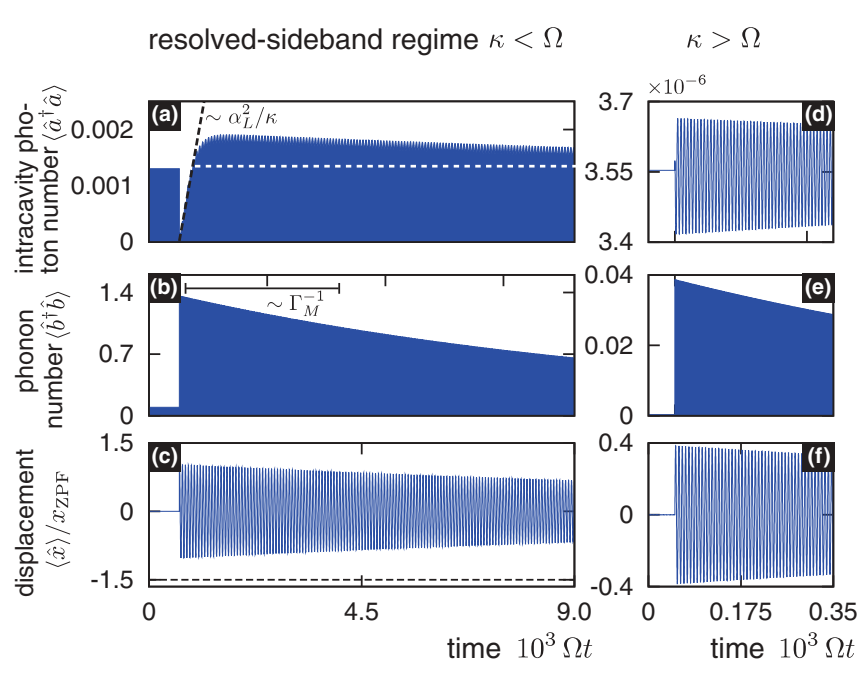

FIG. 2. (Color online) Typical quantum jump trajectories for a weakly driven optomechanical system. These show the expectation values for the photon number (a) and (d), phonon number (b) and (e), and displacement (c) and (f), evolving conditioned on the detection of a photon. The concomitant sudden drop in the radiation pressure force leads to subsequent mechanical oscillations, relaxing during the damping time $\Gamma_{\mathrm{M}}^{-1}$. The dashed line in (c) corresponds to the displacement which would make the optomechanical cavity resonant with the incoming laser. (a)-(c) Sideband-resolved regime ( $\kappa=\Omega / 8)$, with $g_{0} / \kappa=4$. (d)-(f) Bad cavity limit $(\kappa=5 \Omega$, and $\left.g_{0} / \kappa=1 / 10\right)$. Parameters are as follows: detuning $\Delta=\Omega-g_{0}^{2} / \Omega$; coupling $g_{0}=\Omega / 2$; laser drive $\alpha_{L}=5 \times 10^{-3} \Omega$; mechanical damping $\Gamma_{M}=10^{-3} \Omega$; photon decay $\kappa_{I}=\kappa_{O}=\kappa / 2$; bath temperature $T=0$.

antibunching, i.e., $g^{(2)}(0)<1$ [36]. Thus, it is less probable to detect a second photon right after the detection of the first photon. It turns out that we can connect the two-photon correlation function $g^{(2)}(\tau)$ to the intracavity photon number $\left\langle\hat{a}^{\dagger} \hat{a}\right\rangle^{\text {(cond) }}(\tau)$ conditioned on a photon jump at $\tau=0$. This can be done via

$$
g^{(2)}(\tau) \approx \frac{\left\langle\hat{a}^{\dagger} \hat{a}\right\rangle^{(\text {cond })}(\tau)}{\left\langle\hat{a}^{\dagger} \hat{a}\right\rangle\left(\tau=0^{-}\right)} .
$$

Here $\left\langle\hat{a}^{\dagger} \hat{a}\right\rangle\left(\tau=0^{-}\right)$denotes the photon number in any given quantum jump trajectory right before the photon jump [white dashed line in Fig. 2(a)] and $\tau$ denotes the time after the photon jump. The relation of Eq. (9) can be understood intuitively: $\left\langle\hat{a}^{\dagger} \hat{a}\right\rangle^{\text {(cond) }}(\tau)$ is proportional to the probability of detecting a photon conditioned on a photon jump at $\tau=0$. As discussed in Sec. III D, this coincides with the interpretation of $g^{(2)}(\tau)$. The validity of Eq. (9) can be seen more formally by comparing it to the quantum regression formula [32]. For that to hold, we have to require that photon events are rare, which is fulfilled in the regime of weak driving: The photon number entering the definition of the two-photon correlation function (6) is given by a long-time average of the photon number gained from a quantum jump trajectory. Thus, if the jump events are rare, the limiting value $\left\langle\hat{a}^{\dagger} \hat{a}\right\rangle\left(\tau=0^{-}\right)$is assumed most of the time and it will be a good approximation for the long-time average $\left\langle\hat{a}^{\dagger} \hat{a}\right\rangle$ of the photon number. Additionally, the two-photon correlation function $g^{(2)}(\tau)$ can be reconstructed as an ensemble average over many quantum jump trajectories conditioned on a photon 


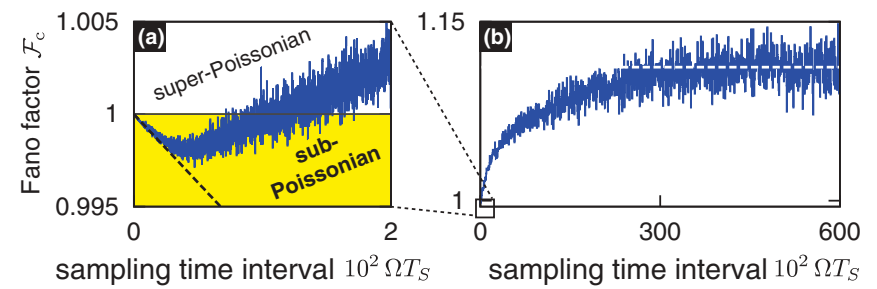

FIG. 3. (Color online) Fano factor $\mathcal{F}_{\mathrm{c}}\left(T_{S}\right)$ for photon detection events, as a function of the sampling time interval $T_{S}$. (a) Fano factor for small sampling times, where antibunching and sub-Poissonian statistics is observed $\left[g^{(2)}(0)<1\right.$ and $\mathcal{F}_{\mathrm{c}}\left(T_{S}\right)<1$; cf. Eq. (7)]. (b) Fano factor for larger sampling times $T_{S}$. We see that $\mathcal{F}_{\mathrm{c}}\left(T_{S} \rightarrow\right.$ $\infty)>1$ saturates and indicates super-Poissonian statistics. This is a characteristical effect of the mechanical motion induced by a photon jump (see main text). Parameters as in Fig. 2(a).

jump at $\tau=0$. Thus, the trajectory depicted in Fig. 2 will be a valid approximation of the ensemble average when $\tau$ is smaller than the average waiting time between photon jumps.

Let us now focus on how the photon detection influences the mechanical degree of freedom: Before observing the single photon, the average radiation pressure force has been very small, since $F_{\text {rad }} \sim\left\langle\hat{a}^{\dagger} \hat{a}\right\rangle$ and $\left\langle\hat{a}^{\dagger} \hat{a}\right\rangle \ll 1$. Now that we know that a photon is detected, however, we deduce that this single photon must have exerted a significant radiation pressure force prior to leaving the cavity. The quantum jump formalism takes this into account in the following way: Upon photon detection, the expectation for the mechanical displacement is updated to reflect the value it must have had due to this relatively large single-photon force, which is much larger than the average force [sudden jump of $\langle\hat{x}\rangle$ in Fig. 2(c) toward a greater displacement]. This reflects photon-phonon entanglement in the state prior to photon detection. After the photon has left the cavity, however, the radiation pressure force becomes very small, since the photon number expectation value $\left\langle\hat{a}^{\dagger} \hat{a}\right\rangle$ now has dropped close to zero. Thus, the displaced mechanical resonator starts to oscillate. This subsequent oscillation decays on the mechanical damping time scale. The photon number itself [and, hence, $g^{(2)}(\tau)$ ] quickly increases again due to the laser driving and then, much more slowly, settles to its limiting value, on a time scale set by $\Gamma_{\mathrm{M}}^{-1}$.

In the following, we focus on the effects of this backaction onto the two-photon correlations for time delays $\tau \neq 0$. As shown in Fig. 2(a), where blue detuning $\Delta>0$ is chosen, the photon number temporarily exceeds its limiting value before settling down. In terms of the two-photon correlation function and Eq. (9), this means that $g^{(2)}(\tau)>1$. This is because the mechanical oscillations bring the cavity closer to resonance. Thus, even though the photon statistics at $\tau=0$ is antibunched, there is the chance of observing super-Poissonian statistics for longer observation times. This can in fact happen, as we will show now. It should be noted that this is another effect where the mechanical motion is crucially important.

As already discussed above, the photon statistics can also be quantified by the Fano factor $\mathcal{F}_{\mathrm{c}}\left(T_{S}\right)$, which is shown in Fig. 3. For the bad cavity case [Figs. 2(d)-2(f)], the Fano factor would be close to the value expected for a simple coherent laser beam, $\mathcal{F}_{\mathrm{c}}(\infty) \approx 1$. This is due to the fact that the photon number conditioned on a photon jump remains very close to its limiting value, with small oscillations [cf. Fig. 2(d)]. Thus, $g^{(2)}(\tau)$ will oscillate around the coherent value 1 as well, such that $\mathcal{F}_{\mathrm{c}}(\infty) \approx 1$; cf. Eq. (7). In contrast, for the strongly coupled and sideband-resolved optomechanical system of Figs. 2(a)-2(c), the Fano factor shows pronounced sub-Poissonian statistics at short times $\left(\mathcal{F}_{\mathrm{c}}<1\right)$, since $g^{(2)}(\tau)<1$ in this case, and super-Poissonian $\left(\mathcal{F}_{\mathrm{c}}>1\right)$ statistics for long times, up to $T_{S} \rightarrow \infty$. Thus, the full counting statistics indicates that typically groups of more than one photon are detected during a single transmission process (before the system settles down again), even if the photons are antibunched at short times.

Let us now briefly discuss the influence of the mechanical bath temperature $T$ onto the resulting photon statistics. To that end, we consider finite temperatures $T$. Note that in our numerical simulations, we choose average thermal phonon numbers $n_{\text {th }}(T) \lesssim 2$ to keep the Hilbert space manageable. These temperatures can be reached by cryogenic cooling if the mechanical frequency is large enough. We find that the photons remain antibunched even for these temperatures, i.e., $g^{(2)}(0)<1$. For the parameters discussed here, the Fano factor in the long-time limit $\mathcal{F}_{c}(\infty)$ decreases with increasing bath temperature. However, the photon statistics remains superPoissonian, i.e., $\mathcal{F}_{c}(\infty)>1$.

In conclusion, we emphasize that the sign of the photon statistics (super-Poissonian vs. sub-Poissonian statistics) can depend on the observation time interval. Thus, $\mathcal{F}_{\mathrm{c}}\left(T_{S}\right)$ has to be analyzed for all times $T_{S}$ to capture the full impact of the optomechanical dynamics on the photon statistics. Additionally, it turns out that these findings are robust against thermal fluctuations (with $n_{\text {th }} \lesssim 2$ ).

\section{B. Cascade of photon transmission at strong driving}

For strong laser driving, we observe an interesting feature: Cascades of transmitted photons. These cascades originate from multiphoton transitions, which appear for red detuning $\Delta=-n g_{0}^{2} / \Omega$ as shown in the level scheme of Fig. 4(a). In this cascade regime, $n>1$ photons enter the cavity simultaneously (involving $n-1$ virtual transitions), since the incidence of $m \neq n$ photons is precluded by energy conservation due to the optomechanically induced photon nonlinearity. To favor the virtual (but resonant) $|0\rangle \rightarrow|n\rangle$ transition over the nonvirtual (but off-resonant) $|0\rangle \rightarrow|1\rangle$ transition, we impose the inequalities

$$
\Gamma_{|0\rangle \rightarrow|n\rangle}>\Gamma_{|0\rangle \rightarrow|1\rangle}, \quad \kappa \ll|\Delta|, \quad \text { and } \quad \kappa \gg \Gamma_{|0\rangle \rightarrow|n\rangle} .
$$

The second and third inequality of Eq. (10) ensure that the intermediate transitions of the resonant transition are virtual, and to prevent heating of the resonator. The transition rates read

$$
\Gamma_{|0\rangle \rightarrow|1\rangle}=\alpha_{L}^{2} \frac{\kappa e^{-\left(g_{0} / \Omega\right)^{2}}}{(n-1)^{2} g_{0}^{4} / \Omega^{2}+\kappa^{2} / 4},
$$

and

$$
\Gamma_{|0\rangle \rightarrow|n\rangle}=\frac{4 \alpha_{L}^{2 n}}{\kappa}\left(\frac{\Omega}{g_{0}^{2}}\right)^{2(n-1)} \frac{e^{-n\left(g_{0} / \Omega\right)^{2}}}{[(n-1) !]^{3}} .
$$

Upon increasing the laser drive, at some point the $n$-photon transition will start to be favored over other processes. 

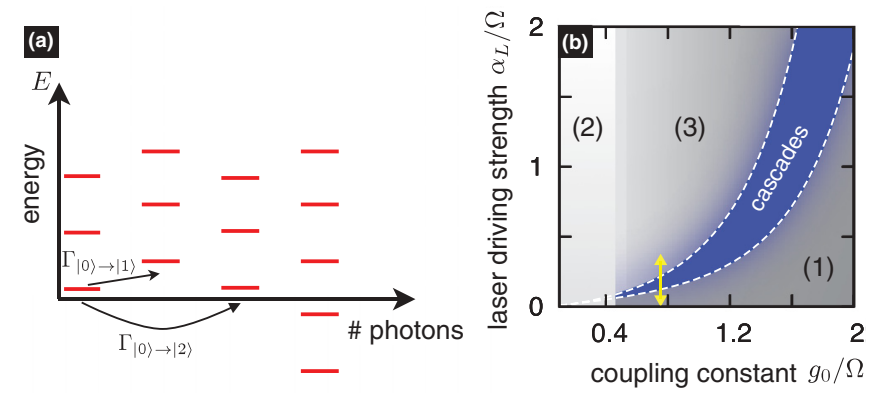

FIG. 4. (Color online) (a) Optomechanical level scheme for a choice of red detuning that enables the two-photon transition. (b) Map of parameters $\alpha_{L} / \Omega$ and $g_{0} / \Omega$, for which a cascade behavior is expected (dark blue region). Note that in regions (1), (2), and (3) the first, second, and third inequality of Eq. (10) is violated. The arrow indicates the trace taken in Fig. 5. Parameters are as follows: $\Delta=-2 g_{0}^{2} / \Omega$ and $g_{0} / \kappa=4$.

Neglecting the mechanical motion for a moment, we expect that these $n$ photons decay out of the cavity on a time scale $\sim \kappa^{-1}$, leading to a photon cascade. In this case, we would naively expect $\mathcal{F}_{\mathrm{c}}^{\text {all }}(\infty) \approx n$. Since we observe the output port only, we expect $\mathcal{F}_{c}(\infty) \approx(n+1) / 2$ for a symmetrical cavity $\left(\kappa_{I}=\kappa_{O}\right)$; cf. Eq. (8). Thus, the Fano factor has to increase when the multiphoton transition starts to become important, regardless of the detector efficiency.

In the following, we will focus on single-photon strong coupling $g_{0} \gtrsim \kappa$. When a specific $n$-photon resonance is chosen and for a fixed ratio $g_{0} / \kappa$, the laser driving strength $\alpha_{L} / \Omega$ and the coupling strength $g_{0} / \Omega$ is varied. A map of these two parameters is shown in Fig. 4(b), where the cascade regime is highlighted.

In Fig. 5(a), the Fano factor $\mathcal{F}_{\mathrm{c}}(\infty)$ is shown as a function of the laser drive. We see that $\mathcal{F}_{\mathrm{c}}(\infty) \approx 1$ for weak laser driving (Poisson statistics), while $\mathcal{F}_{\mathrm{c}}(\infty)$ increases rapidly beyond the threshold of the laser drive predicted by the above analysis. There, the $n$-photon transition is favored and photons are emitted in bunches. In contrast to our naive expectation, $\mathcal{F}_{\mathrm{c}}(\infty)$ is larger than $(n+1) / 2$ and, hence, $\mathcal{F}_{c}^{\text {all }}(\infty)>n$. This fact is due to the mechanical motion induced by photon detection events (cf. Fig. 6) where a typical jump trajectory in the cascade regime is displayed.

Upon detection of a photon leaving the cavity, the expectation value of the intracavity photon number increases; cf. Fig. 6(a). This behavior is known for situations with photon bunching [36,41], and it arises because the detection of a photon is most likely due to a previous multiphoton transition $[36,41]$. Thus, upon observing one photon leaving the cavity, we can now reasonably expect that there are still more photons inside the cavity. These remaining photons are likely to be emitted rapidly afterwards (the rate for $n$ photons to decay is $n \kappa)$. However, as discussed above in our analysis of the weak-driving case, the mechanical resonator gets displaced by a photon jump and starts to oscillate subsequently [cf. Figs. 6(b) and 6(c)]. This mechanical motion can bring the cavity into resonance [cf. Fig. 6(c)], which then allows for the transmission of additional photons. In this way, cascades of more than $n$ transmitted photons become possible [i.e., $\left.\mathcal{F}_{c}^{\text {all }}(\infty)>n\right]$. Note that once a single cascade of photon
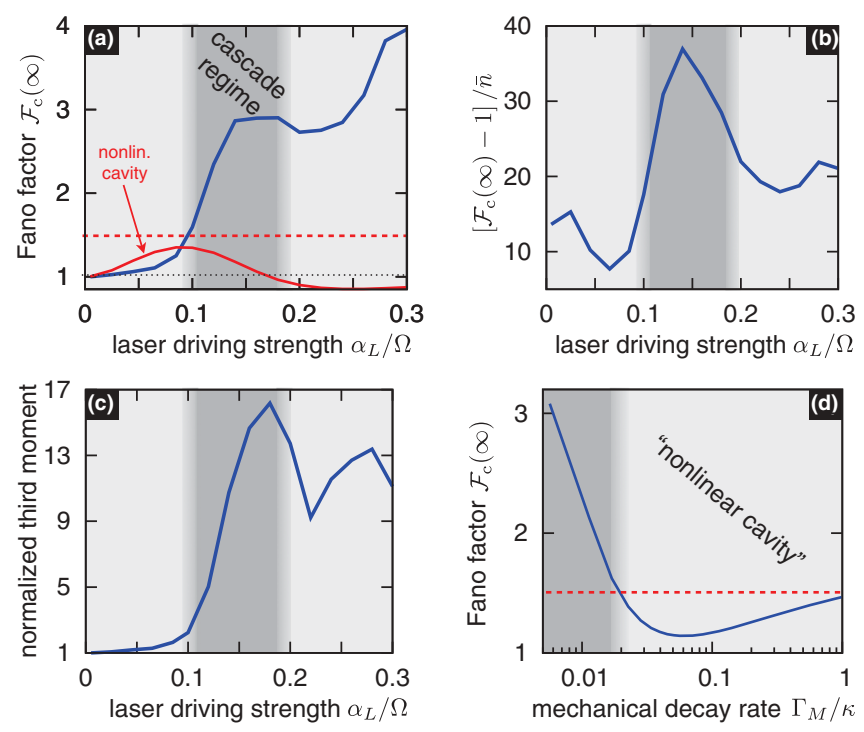

FIG. 5. (Color online) Photon statistics in the "photon cascade regime." (a) Fano factor $\mathcal{F}_{c}(\infty)$ as a function of laser drive. The red, dashed line represents the naive expectation $\mathcal{F}_{c}(\infty)=(n+1) / 2(=$ 1.5). In the highlighted region, the multiphoton transition is favored [cf. Fig. 4(b)]. At the onset of this regime, the Fano factor $\mathcal{F}_{\mathrm{c}}$ rises beyond the naive expectation. The red curve represents $\mathcal{F}_{c}(\infty)$ for a nonlinear cavity (Kerr nonlinearity) with the same parameters. (b) Rescaled deviation from Poisson statistics. (c) Normalized third moment, $\left\langle(N-\langle N\rangle)^{3}\right\rangle /\langle N\rangle$. (d) Fano factor as a function of mechanical decay rate $\Gamma_{M}$, approaching the value of a nonlinear cavity for large $\Gamma_{M}$. Parameters (a)-(c) are as follows: $\Delta=-2 g_{0}^{2} / \Omega$, $g_{0}=\Omega / \sqrt{2}, \kappa=g_{0} / 4=\Omega /(4 \sqrt{2}), \Gamma_{M}=10^{-3} \Omega, T=0$. (d) Same parameters, but $\alpha_{L}=0.15 \Omega$.

transmission terminates, no photon jumps are observed any more and the mechanical displacement is able to relax towards its equilibrium value.

Let us now focus on even larger laser driving strength. In this case, the third inequality of Eq. (10) is violated, and the average photon and phonon numbers as well as $\mathcal{F}_{\mathrm{c}}(\infty)$ increase even further. Additionally, no clear cascades are observable anymore. The strong photon noise in that case can be explained as being due to the transmission modulated stochastically by a fluctuating mirror, whose motion has been heated. This is seen in a decrease of the rescaled Fano factor $\left(\mathcal{F}_{\mathrm{c}}(\infty)-1\right) / \bar{n}$ [Fig. 5(b)]: The rescaling is introduced to be able to distinguish two different regimes in which the Fano factor tends to be large but where the fluctuations are of qualitatively different origin. In the photon cascade regime, the increase of the Fano factor is due to large and long-time intracavity photon correlations. Since the rescaled Fano factor is maximal in this regime, the time-averaged intracavity photon correlations are strongest; cf. Eq. (7). As we have seen, the Fano factor increases further for even larger laser driving strengths. By inspecting the rescaled Fano factor we observe, however, that the time-averaged intracavity photon correlations decrease. Thus, the Fano factor in this case increases further due to an increased photon transmission rate and not because of large intracavity photon correlations.

Note that the statistical data obtained from the quantum jump trajectories also provide access to higher moments. The 

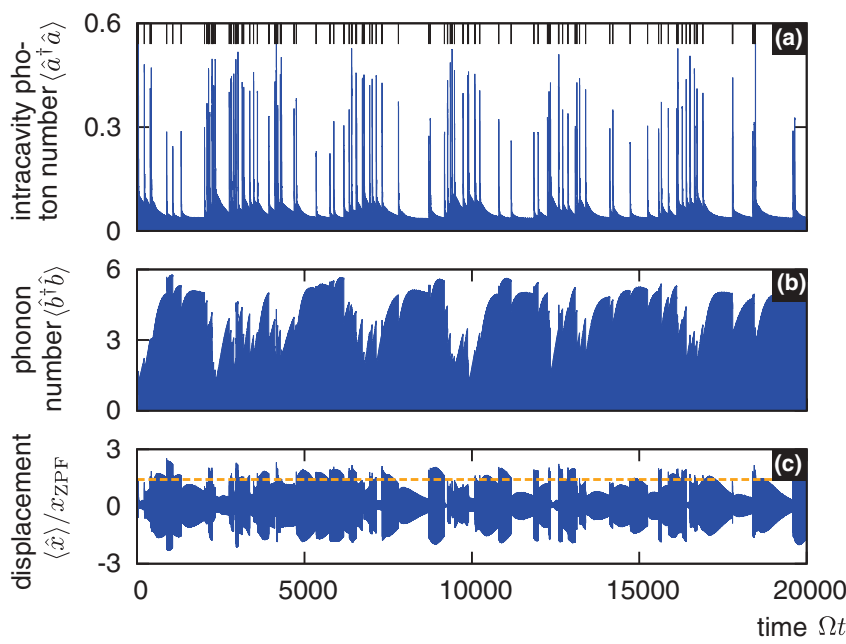

FIG. 6. (Color online) Typical quantum jump trajectory in the cascade regime. The pattern of vertical lines in (a) indicates the actual photon jump events, where bunching is clearly observable. (b) Phonon number expectation value. If a photon jump occurs, the mechanical state gets updated, leading to an instantaneous change of the phonon number. This reflects photon-phonon entanglement. The orange, dashed line in (c) corresponds to the displacement that would make the cavity resonant with the incoming laser. Parameters as in Fig. 5(a), and $\alpha_{L}=0.15 \Omega$.

third moment [Fig. 5(c)], for example, displays a maximum in the cascade regime studied here.

Let us now discuss two other pieces of evidence which show that the enhanced cascade of photon transmission is due to the presence of the mechanical resonator. First, we compare our optomechanical system to a nonlinear cavity (Kerr nonlinearity). There, we find that $n$-photon transitions are observable as well; cf. red curve in Fig. 5(a). However, in contrast to the optomechanical system, the Fano factor in that case does not exceed the naively expected value $\mathcal{F}_{c}(\infty) \approx(n+1) / 2$.

Second, as another indication of the strong mechanical signature in the photon correlations, we note that the Fano factor depends strongly on the mechanical damping rate [Fig. 5(d)]. For smaller damping, the mechanical resonator oscillates more often through the cavity resonance such that even more photons are transmitted, and the Fano factor increases. On the other hand, by choosing $\Gamma_{M} \sim \kappa$, the limit of a nonlinear cavity can be achieved. Experimentally, $\Gamma_{M}$ could be tuned by making use of the optomechanical cooling effect $[42,43]$ using a second optical mode.

Let us now briefly discuss a higher resonance condition, with $n>2$; cf. Fig. 7(a). In general, the minimum laser driving strength needed to generate photon cascades increases with $n$. This is because $n$ photons enter the cavity simultaneously via $n-1$ virtual transitions. Furthermore, we observe the same enhanced cascade behavior as for $n=2$ : First, we see that the Fano factor rises strongly for sufficiently large laser driving, as it was the case for $n=2$. Second, as already discussed before, we can compare the optomechanical system to a nonlinear cavity (Kerr nonlinearity). We observe that the Fano factor for the Kerr cavity lies below the optomechanical Fano factor.
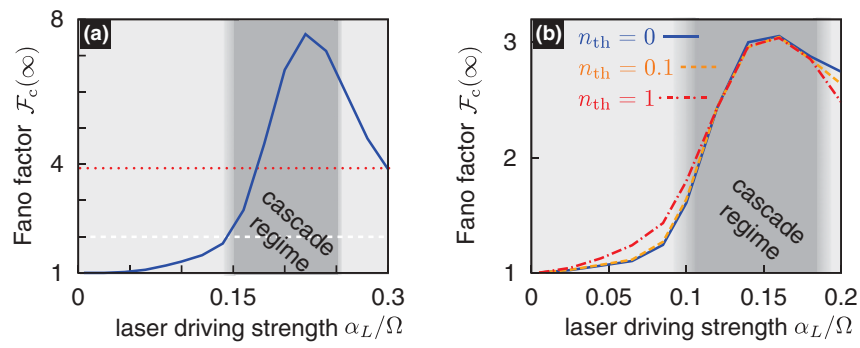

FIG. 7. (Color online) (a) Fano factor $\mathcal{F}_{c}(\infty)$ for a higher resonance $(n=3)$ showing photon cascades. The white dashed line shows the naive expectation $\mathcal{F}_{c}(\infty)=(n+1) / 2$. The red, dotted line indicates the maximum value of $\mathcal{F}_{c}(\infty)$ which is reached by a nonlinear cavity (Kerr nonlinearity) with the same parameters. (b) Fano factor $\mathcal{F}_{c}(\infty)$ for $n=2$ and different temperatures. Parameters are as follows: (a) $n=3, \Delta=-3 g_{0}^{2} / \Omega, g_{0}=\Omega / 2, g_{0} / \kappa=4$, $T=0$. (b) Same parameters as in Fig. 5, but $n_{\text {th }}=0$ (solid blue line), $n_{\mathrm{th}}=0.1$ (dashed orange line), and $n_{\mathrm{th}}=1$ (dash-dotted red line).

Thus, we infer that photon transmission is strongly enhanced by the mechanical resonator for $n>2$ as well.

Finally, in the following, we will discuss the influence of the mechanical bath temperature $T$ onto the photon cascades (taking the case $n=2$ for concreteness). We consider the Fano factor $\mathcal{F}_{c}(\infty)$ for different laser driving strengths and different temperatures; cf. Fig. 7(b). Let us first focus on laser driving strengths $\alpha_{L}$ lying below the cascade regime. There, we find that the Fano factor increases with increasing temperature for fixed $\alpha_{L}$. This is because the thermal fluctuations of the position of the cantilever allow for the stochastic transmission of photons, which leads to an increase of $\mathcal{F}_{c}(\infty)$. In the photon cascade regime, however, we observe that the Fano factor does not change strongly with increasing temperature. This is because for strong laser driving many phonons are generated by the coherent dynamics even for zero temperature; cf. Fig. 6(b). Thus, in contrast to the weak driving case discussed above, the few thermal phonons do not affect the dynamics of the mechanical resonator considerably.

\section{CONCLUSION}

We have analyzed the full statistics of photons transmitted through an optomechanical system. The photon correlations crucially depend on the observation time and may even change from sub-Poissonian to super-Poissonian statistics in the longtime limit. For larger laser driving, photon cascades may be observed. These contain clear signatures of the strong lightmechanics coupling, and the influence of the mechanical motion could even be tuned by varying the mechanical decay rate.

Note added in proof. Recently, we became aware of a related paper by $\mathrm{Xu}, \mathrm{Li}$, and Liu [44].

\section{ACKNOWLEDGMENTS}

Financial support from the Defense Advanced Research Projects Agency ORCHID, the Emmy-Noether program, and the European Research Council is gratefully acknowledged. A.K. thanks the High Performance Computing group at the Friedrich-Alexander-Universität Erlangen-Nürnberg for stimulating discussions. 
[1] K. M. Birnbaum et al., Nature (London) 436, 87 (2005).

[2] A. Kubanek, A. Ourjoumtsev, I. Schuster, M. Koch, P. W. H. Pinkse, K. Murr, and G. Rempe, Phys. Rev. Lett. 101, 203602 (2008).

[3] A. Majumdar, M. Bajcsy, and J. Vučković, Phys. Rev. A 85, 041801 (2012).

[4] A. Reinhard et al., Nature Photonics 6, 93 (2012).

[5] P. Münstermann, T. Fischer, P. Maunz, P. W. H. Pinkse, and G. Rempe, Phys. Rev. Lett. 82, 3791 (1999).

[6] M. L. Terraciano et al., Nature Physics 5, 480 (2009).

[7] C. J. Hood et al., Science 287, 1447 (2000).

[8] A. C. Doherty, T. W. Lynn, C. J. Hood, and H. J. Kimble, Phys. Rev. A 63, 013401 (2000).

[9] D. Rotter et al., New J. Phys. 10, 043011 (2008).

[10] For reviews see, e.g., T. J. Kippenberg and K. J. Vahala, Science 321, 1172 (2008); F. Marquardt and S. M. Girvin, Physics 2, 40 (2009).

[11] J. D. Teufel et al., Nature (London) 475, 359 (2011).

[12] J. Chan et al., Nature (London) 478, 89 (2011).

[13] K. W. Murch et al., Nat. Phys. 4, 561 (2008).

[14] F. Brennecke et al., Science 322, 235 (2008).

[15] E. Verhagen et al., Nature (London) 482, 63 (2012).

[16] J. Chan et al., Appl. Phys. Lett. 101, 081115 (2012).

[17] M. Ludwig, B. Kubala, and F. Marquardt, New J. Phys. 10, 095013 (2008).

[18] P. Rabl, Phys. Rev. Lett. 107, 063601 (2011).

[19] A. Nunnenkamp, K. Børkje, and S. M. Girvin, Phys. Rev. Lett. 107, 063602 (2011).

[20] J. Qian et al., Phys. Rev. Lett. 109, 253601, (2012).

[21] S. Mancini, V. I. Man'ko, and P. Tombesi, Phys. Rev. A 55, 3042 (1997).

[22] S. Bose, K. Jacobs, and P. L. Knight, Phys. Rev. A 56, 4175 (1997).

[23] A. Nunnenkamp, K. Børkje, and S. M. Girvin, Phys. Rev. A 85, 051803 (2012).
[24] G. -F. Xu and C. K. Law, arXiv:1211.5445.

[25] M. Ludwig, A. H. Safavi-Naeini, O. Painter, and F. Marquardt, Phys. Rev. Lett. 109, 063601 (2012).

[26] K. Stannigel, P. Komar, S. J. M. Habraken, S. D. Bennett, M. D. Lukin, P. Zoller, and P. Rabl, Phys. Rev. Lett. 109, 013603 (2012).

[27] C. Fabre, M. Pinard, S. Bourzeix, A. Heidmann, E. Giacobino, and S. Reynaud, Phys. Rev. A 49, 1337 (1994).

[28] S. Mancini and P. Tombesi, Phys. Rev. A 49, 4055 (1994).

[29] C. K. Law, Phys. Rev. A 51, 2537 (1995).

[30] J. Dalibard, Y. Castin, and K. Mølmer, Phys. Rev. Lett. 68, 580 (1992).

[31] C. W. Gardiner, A. S. Parkins, and P. Zoller, Phys. Rev. A 46, 4363 (1992).

[32] H. Carmichael, An Open Systems Approach to Quantum Optics (Springer, Berlin, 1993).

[33] M. B. Plenio and P. L. Knight, Rev. Mod. Phys. 70, 101 (1998).

[34] H. M. Wiseman and G. J. Milburn, Phys. Rev. A 47, 1652 (1993).

[35] A. A. Gangat, T. M. Stace, and G. J. Milburn, New J. Phys. 13, 043024 (2011).

[36] M. Ueda, N. Imoto, and T. Ogawa, Phys. Rev. A 41, 3891 (1990).

[37] D. F. Walls and G. J. Milburn, Quantum Optics (Springer, New York, 1994).

[38] L. Mandel and E. Wolf, Optical Coherence and Quantum Optics (Cambridge University Press, Cambridge, 1995).

[39] S. Singh, Opt. Commun. 44, 254 (1983).

[40] X. T. Zou and L. Mandel, Phys. Rev. A 41, 475 (1990).

[41] V. Parigi et al., Science 317, 1890 (2007).

[42] F. Marquardt, J. P. Chen, A. A. Clerk, and S. M. Girvin, Phys. Rev. Lett. 99, 093902 (2007).

[43] I. Wilson-Rae, N. Nooshi, W. Zwerger, and T. J. Kippenberg, Phys. Rev. Lett. 99, 093901 (2007).

[44] X.-W. Xu, Y.-J. Li, and Y.-X. Liu, arXiv:1212.4221 [Phys. Rev. A (to be published)]. 